\title{
La thématique de l'aliénation dans The Changeling
}

André Bry

\section{(2) OpenEdition \\ Journals}

\section{Édition électronique}

URL : http://journals.openedition.org/shakespeare/1314

DOI : $10.4000 /$ shakespeare.1314

ISSN : 2271-6424

Éditeur

Société Française Shakespeare

Édition imprimée

Date de publication : 1 novembre 1989

Pagination : 85-94

Référence électronique

André Bry, "La thématique de l'aliénation dans The Changeling ", Actes des congrès de la Société française Shakespeare [En ligne], 7| 1989, mis en ligne le 01 janvier 2007, consulté le 21 avril 2019. URL : http://journals.openedition.org/shakespeare/1314; DOI : 10.4000/shakespeare.1314 


\title{
Société Française Shakespeare
}

\author{
Actes des Congrès \\ 1985 - 1986 - 1987
}

\section{LA FOLIE}

\section{SHAKESPEARE ET LES ARTS \\ LE TRAGIQUE}




\section{LA THEMATIQUE DE L'ALIENATION DANS THE CHANGELING.}

\section{A. BRY}

Les études qui ont été faites depuis un demi-siècle sur la structure du Changeling ont montré qu'il existait chez les auteurs de cette pièce une volonté consciente de faire ressortir, entre les deux intrigues qui se déroulent parallèlement, tout un jeu de correspondances verbales et thématiques qui lui donne une cohérence structurelle particulière. On a remarqué notamment que si l'asile de fous est le lieu privilégié où les formes les plus évidentes du dérèglement mental se donnaient libre cours, ce qui se passe à l'extérieur de ce lieu d'enfermement révèle d'autres formes, moins évidentes à première vue, mais en fait encore plus dangereuses, d'une véritable déraison. Mais la place qu'occupe l'aliénation mentale dans la pièce ne pourrait nous autoriser à parler, à propos du Changeling, d'une véritable thématique de l'aliénation, si d'autres formes d'aliénation, familières au lecteur moderne, ne pouvaient se déceler dans ce drame vieux de 250 ans, et où le mot lui-même n'apparaît même pas, bien qu'il existât déjà dans certaines de ses acceptions actuelles.

Avant de tenter de démontrer l'intérêt d'une telle approche, il convient de rappeler tout ce que ce mot peut suggérer au lecteur moderne. Outre les diverses formes d'aliénation mentale, à laquelle se rattache la passion amoureuse, dans la mesure où celle-ci provoque une altération, une dégradation du moi, nous savons que ce mot peut évoquer, dans le domaine juridique, l'aliénation d'un bien à titre gratuit ou onéreux, et par extension, la perte ou l'abandon de tout bien naturel : on parle d'aliénation de liberté ou d'indépendance. Un autre sens, très ancien lui aussi, en anglais comme en français, évoque une perte affective, l'éloignement, la froideur, l'hostilité ; on parle de l'aliénation des esprits et des sentiments, et l'exemple le plus ancien d'emploi de ce terme en anglais, d'après l'O.E.D., traduit l'idée d'éloignement à l'égard de Dieu (1388). On voit que par un glissement de sens on passe du domaine des relations personnelles au domaine religieux et au domaine moral : on en est venu à parler d'aliénation pour évoquer tout processus par lequel l'homme devient étranger à ce qui constitue son essence et perd la claire conscience de ses rapports avec l'Autre, que ceci soit dû ou non aux conditions sociales ou économi- 
ques, comme c'est le cas dans l'aliénation selon Marx ; on remarque que l'acception marxiste est aussi assez proche de l'acception juridique. Toutes ces significations diverses ont en effet un point commun : l'aliénation implique une perte, une dégradation, consciente ou inconsciente, provisoire ou définitive.

Les aliénés enfermés chez le Docteur Alibius occupent dans la pièce une place très réduite. Vers la fin du permier acte, on les entend, mais on ne les voit pas : l'édition originale porte cette indication scénique : «Madmen within»; ils restent en coulisse et leur rôle ne comporte que quatre courtes phrases. Dans l'Acte III, Scène III, leurs cris incitent par deux fois Lollio à quitter la scène, ce qui permet à Antonio de dévoiler sa passion pour Isabella, et au gardien de surprendre son manège. Déguisés en oiseaux et en animaux divers, ils apparaissent un instant "above", sur la scène supérieure du thêatre élisabéthain, et y restent juste assez pour qu'Isabella puisse commenter leur présence en sept vers avant le retour de Lollio, retour qui implique que l'ordre ayant été rétabli, les fous ont regagné leurs quartiers. A la fin de l'Acte IV, une autre indication scénique : «The Madmen and Fools dance», signale leur dernière apparition sur la scène, sans indiquer la durée de cet étrange ballet.

Et pourtant leur importance est plus grande qu'il n'y paraît. Ces quelques êtres sans nom et sans visage, dont on ignore le nombre et l'identité comme ils l'ignorent eux-mêmes, ont un langage dépourvu de sens, à peine plus élaboré qu'un cri, mais qui trahit d'abord le désir le plus élémentaire : la faim*; ils constituent dans l'univers de la pièce un symbole grotesque et effrayant. Incapables de raisonner et impossibles à raisonner, ils n'obéissent qu'à la menace du fouet que brandit leur gardien Lollio, prompt à renvoyer au «chenil» (v. 91) un Francisco jugé "dangereux». Lollio aime faire étalage de son autorité («You see how I awe my flock; a shepherd has not his dog at more obedience») - autorité sur ceux qu'il compare à des chiens : mais, s'il est sûr de lui quand il a affaire à Franciscus, un simulateur, il lui arrive d'être inquiet, mal assuré de son pouvoir : il craint d'être débordé, non par le troupeau docile des idiots, mais par ces "scholars of the upper room", si souvent "out of order» (III, iii, 10) et dont l'agressivité, déchaînée, pourrait devenir redoutable : «Again? Must I come amongst you? Would my master were come home! I am not able to govern both these wards together (III, iii, 166). Leur irruption sur la scène (Madmen above, some as birds, others as beasts) permet à Isabella de nous les décrire, à nous simples lecteurs, et de tenter de faire comprendre à Antonio que le miroir qu'ils tendent au reste de l'humanité doit lui inspirer de la crainte :

* «They must be fed», dit Alibius vers la fin du premier acte, et le langage de Franciscus traduit en la parodiant cette préoccupation des vrais fous : «Thou feedst thy horseswell, they shall obey thees (III, iii, 55) 


\begin{abstract}
ANT. What are these?
ISA. Offear enough to part us ;

Yet are they but our schools of lunatics,

That act their fantasies in any shapes

Suiting their present thoughts ; if sad, they cry ;

If mirth be their conceit, they laugh again ;

Sometimes they imitate the beasts and birds,

Singing, or howling, braying, barking ; all

As their wild fancies prompt' em.
\end{abstract}

(III, iii, 191-8)

Toutefois, ce spectacle lui inspire aussi de la pitié : «tis too full of pity / To be laugh' d at « (43-44). Leurs déguisements pittoresques, qu' ils revêtent sans doute quand leur singulier thérapeute exploite leur maladie en les produisant en public, ne les transforment pas seulement en acteurs d'un antimasque grotesque ; ils les font apparaître tels qu'ils sont en fait devenus ; comme l'écrit M.C. Bradbrook, «The chorus of madmen depict the bestial element in man, rather as Caliban does, or the rout in Comus... They are a symbolic presentation of evil». De ce mal qu'ils représentent, de ce retour à l'animalité qu' ils nous montrent, on peut les imaginer personnellement irresponsables. La position des juristes élisabéthains en la matière était plus humaine qu'on ne peut l'imaginer, à voir la façon dont on traitait les aliénés à Bedlam et autres enfers analogues, puisque les crimes commis sous l'empire d'une folie reconnue n'étaient pas considérés comme punissables. Et pourtant, un doute surgit à la fin du premier acte, lorsque Lollio nous apprend que le Gallois dont nous entendons les plaintes délirantes est un incurable «undone by a mouse, that spoil' $d$ him a parmesant; lost his wits for' t». Cette plaisanterie facile à l'égard des Gallois, réputés pour leur goût immodéré du fromage, suggère sans doute aussi que d'autres appétits que celui de la gourmandise peuvent faire de nous des fous inguérissables ; ici comme dans le langage de De Flores - semblable sur ce point à celui de maint personnage shakespearien - la nourriture pourrait servir de métaphore à la sexualité : la pièce ne nous montre-t-elle pas que de tous les péchés capitaux, le plus répandu, le plus redoutable, le plus aliénant, c'est la luxure ?

Isolée de son contexte, l'aventure d'Antonio et de Franciscus n'est qu'une ruse de comédie bien traditionnelle: deux jeunes galants déguisés trompent la vigilance d'un barbon jaloux ; mais dans le Changeling, cet épisode semble aussi destinéà nous rappeler qu'il est dangereux de simuler la folie ou la faiblesse d'esprit quand cette entreprise a pour but de détruire un mariage - si mal assorti qu'il puisse être. Les deux jeunes gens en sont réduits, pour tenter de séduire l'objet de leur désir, à se feindre autres que ce qu'ils sont, courant ainsi le risque que le masque ne leur colle trop bien au visage, comme le découvre un jour le Lorenzaccio de Musset ; le 
travestissement qu'ils s'imposent, qu'il s'agisse du pseudo-délire logique et satirique d'Antonio, ou de la fureur poétique, elle aussi pleine de méthode, que cherche à feindre Franciscus, entré chez Alibius pour cause de folie amoureuse (III, iii, 45), a quelque chose de dégradant pour quiconque a le respect de soi-même; de leur propre aveu (V, iii, 204-9), leur simulation n'a été que sottise et folie, moins parce qu'elle a échoué que parce qu'elle aurait pu réussir ; à vouloir corrompre une honnête femme, on risque de se retrouver «in hell», comme Beatrice et De Flores au terme de leur sinistre partie de «barley-break». Mais, pas plus que le langage poétique et obscur de Franciscus, qui s'efforce d'être en même temps «The lunatic, the lover and the poet» dont parle Thésée dans A Midsummer Night's Dream, le discours pétrarquisant d'Antonio n'a de prise sur Isabella. A ce discours, qui prête à sa beauté le pouvoir de métamorphoser celui quil'aime (idée sans doute empruntée à la scène de séduction de Volpone), elle répond en se travestissant en une réplique féminine de Franciscus, en une caricature de caricature. Il en faut plus pour guérir les deux soupirants ; leurs symptômes vont même s'aggraver quand Lollio, révélant à chacun la présence de l'autre, suscite en eux une folie meurtrière; les voilà métamorphosés en chevaliers grotesques, prêts à s'entretuer pour obtenir les faveurs d'une Dame qui se joue d'eux. Il faudra qu'au 5e acte leur imposture soit dévoilée, et qu'ils échappent de peu à la fureur vengeresse de Tomazo pour qu'ils prennent enfin conscience de la dangereuse futilité de leur dessein, et que dans les derniers vers ils renoncent à la folie, à la sottise. Ridiculisés et démasqués, ils comprennent ce qu'il y avait de réel dans leur aliénation simulée, et s'en libèrent par une renonciation spontanée.

Dans ses rapports avec les aliénés, réels ou simulés, le personnage de Lollio nous apparait très ambigu, puisqu'il est tantôt le tyran bouffon mais sûr de lui, tantôt le reflet pitoyable des obsessions de ses "élèves", tantôt le subalterne accablé par sa charge. Les simulateurs se moquent de lui comme il se moque de son maître et dans cet asile la folie des uns et la sottise des autres se démasquent mutuellement, parfois avec l'aide d'Isabella. A certains égards, Lollio tient du bouffon shakespearien dans la mesure où il est à la fois un simple d'esprit tourmenté par le désir et le dépositaire, à l'occasion, d'une sagesse authentique. Cette sagesse, il ne faut certes mieux pas la chercher dans les tests d'intelligence qu'il impose à ses patients; mais il est significatif que ce soit Lollio qui soit chargé, au début de III, iii, de rappeler l'universalité de la folie et de la sottise : «Here's none here but fools and madmen», se lamente Isabella, et Lollio lui répond: «Where will you find any other if you should go abroad ?» Placé au milieu même de la pièce, ce lieu commun traditionnel en résume le déroulement tout entier. Psychose incurable ou égarement passager du cœur et de l'esprit, l'hôpital des fous ne renferme rien qui soit bien dangereux ; c'est un miroir terni où se reflètent à peine l'aveuglement, la folie destructrice de ceux qui ont la présomption de croire en la solidité de leur jugement, et que la passion incite à commettre les pires folies, les pires sottises.

Alonzo meurt poignardé sans seulement savoir pourquoi. Si l'assassin prenait le temps de le lui dire, croirait-il que celle dont il porte au doigt le gage 
d'amour a ordonné sa mort, lui qui ressentait comme autant d'insultes les conseils de prudence que lui donnait son frère Tomazo, surpris de l'accueil glacial et muet que Beatrice réserve à son fiancé ? Tomazo discerne à juste titre chez son frère un mal qu'il appelle "Love" s tame madness» (à la fin de II, i). Mais, à vrai dire, la folie d'amour, telle qu'elle nous est décrite dans la pièce, n'est pas toujours si facilement maîtrisable.

La première victime en est Alsemero; dès la première scène, en quête d'heureux présages et malgré tout inquiet de ce que le destin lui réserve s'il $s$ 'abandonne, il renonce à tous ses projets pour courtiser une jeune fille dont il ne sait rien sinon qu'elle est belle ; surpris de ne plus très bien savoir qui il est, où il est, il se demande si «there be some hidden malady Within me, that I understand not». Cette "maladie" va l'inciter à rester au lieu de fuir - sa première impulsion quand il apprend que Beatrice est fiancée - et à projeter un duel avec le rival qui fait obstacle à son bonheur. Mais chez Alsemero, la passion amoureuse reste limitée ; lorsqu'elle entre en conflit avec son sentiment de l'honneur, elle cède la place ; mais c'est pour qu'apparaisse alors une autre forme d'aliénation, comme nous le verrons plus loin.

Chez De Flores, qui va savoir mieux que lui prendre la place d'Alonzo, l'aliénation amoureuse prend une forme différente : nulle trace d'aveuglement sur la nature de son désir n'apparaît chez lui ; il sait que l'amour est une attirance purement physique comme la faim, mais aussi difficile à dominer : «I know she hates me / Yet cannot choose but love her» (II, i, 234-5). Pour assouvir ce désir de la posséder qui le possède, il va en toute lucidité jusqu'au meurtre, au sucide et à l'enfer («now we' re left in hell», V, iii, 163).

Mais c'est dans le personnage de Beatrice que s'incarne le plus visiblement cette forme d'aliénation par l'amour qui implique l'obscurcissement de l'intelligence, la disparition du sens moral et la perte du sentiment de la réalité. Les dramaturges ont souligné ce phénomène en prêtant à leur héroïne un discours plein d'une sagesse apparente. Dans I, i, 72-76 comme dans II, i, 6-19, Beatrice ne fait l'éloge de la raison, du jugement, chez elle-même ou chez autrui, que pour en démentir, aussitôt après, le pouvoir sur son propre comportement. Elle admet que quand elle s'est fiancée «sure my eyes were mistaken» $(\mathrm{I}, \mathrm{i}, 84)$; mais elle n'envisage pas un seul instant, avant de se livrer à une nouvelle passion, fondée comme la première sur ce que les yeux seuls lui révèlent, de mettre en question la sûreté de son jugement. Ce qui fait de Beatrice une fiancée parjure puis une épouse infidèle, c'est l'asservissement au désir du moment, qui l'incite notamment à confier à un homme dont elle a instinctivement peur le soin de tuer son fiancé pour qu'elle puisse épouser un autre homme en toute tranquillité. C'est en poignardant Alonzo, bien plus encore qu'en réclamant sa virginité pour salaire, que De Flores se fait le "meurtrier de son honneur". En utilisant cette formule (III, iv, 122), Beatrice trahit un terrible aveuglement moral. Elle nous apparaît comme un animal à peine conscient de sa férocité naturelle. Elle ne comprendra que trop tard qu'elle a aliéné son âme. Même si, comme le veut la logique de la pièce, elle est vouée au même destin que De Flores, elle semble parfois aussi proche des patients d'Alibius que de son complice dans le 
crime.

Chacun sait qu'en vertu d'une coutume qui semblait naturelle à l'époque où fut écrit The Changeling, les jeunes filles et les femmes mariées ne jouissaient pas de la même liberté que les hommes; pères et époux, auxquels les lois accordaient des pouvoirs exorbitants, se faisaient un devoir de les protéger d'elles-mêmes, de veiller à ce qu'elles ne deviennent pas les victimes de la "fragilité" qu'on leur attribuait. Ces préoccupations morales cachaient trop souvent un sentiment moins avouable : celui de la propriété. L'attitude d'Alibius estàcet égard exemplaire : pour protéger sa jeune épouse contre la tentation du dévergondage, il confie à Lollio, le gardien de ses pensionnaires, le soin de la surveiller - et en fait de la séquestrer. Il est normal qu'Isabella ressente comme un affront cette volonté de la parquer, de la mettre en cage - elle utilise les mots "cage" et "pinfold" au début de III, iii - mais elle fait face à cette situation avec une sagesse méritoire. Il ne lui faut pas beaucoup de temps pour découvrir que le geôlier choisi par son mari trahirait volontiers son maître, et que deux galants se sont introduits dans l'asile qui lui sert de prison dans le but de la séduire. Elle savoure l'ironie de la situation et, tout en exprimant apparemment la pensée des dramaturges, elle en tire une morale qui pourrait sortir de L'Ecole des Femmes :

would a woman stray,

She need not gad abroad to seek her sin, It would be brought home one ways or other :

(213-215).

Plutôt que de chercher dans l'adultère, qui d'ailleurs la mettrait à la merci de Lollio, le chemin de la liberté, elle révèle à son mari, dans la dernière scène, la vanité des précautions qu'il a prises : «You are a jealous coxcomb, keep schools of folly, / And teach your scholars how to break your own head» (V, iii, 211-212). Le dénouement laisse espérer qu'Isabella va jouir de la liberté qu'elle a conquise et dont elle est digne ; elle qui a su échapper à la tentation de se libérer d'une forme d'aliénation pour tomber dans une autre. Elle aura au moins la liberté accordée aux Anglaises au début du XVIIe siècle, qui étonnait alors les voyageurs étrangers...

On est tenté d'établir un parallèle entre Isabella et Beatrice en tant que victimes de l'oppression masculine. Si nous analysons les rapports de cette dernière avec son père, nous remarquons que contrairement aux sources utilisées par les dramaturges, le texte du Changeling n'indique pas que Vermandero ait recouru à la violence ou à la menace pour obtenir le consentement de sa fille à un mariage dont il est toutefois enchanté, car il considère Alonzo, " $a$ gallant and a courtier", comme un parti extrêmement flatteur et avantageux. Beatrice, en ce qui la concerne, s'estime victime de "the command of parents" : c'est ce qu'elle dit à Alsemero (II, ii, 20), et un peu plus loin dans la même scène, s'adressant à De Flores, elle dit son regret de n'être pas née homme : «I should not then be forc' d to marry one / I hate beyond all 
depths» (110-111). Mais quand elle s'exprime en aparté, elle nous dit: «sure my eyes were mistaken, I This was the man was meant me» (I, $\mathrm{i}, 84-85)$, ou encore : «I shall change my saint, I fear me» (155), formules qui indiquent qu'elle s'est fiancée de son plein gré, si entre temps son cœur a changé. C'est de cette inconstance que De Flores tire parti dans leur confrontation de III, iv, quand il s'ecrie : «thou whore in thy affection» (142). Plus encore que son père, tel qu'il est, Beatrice a peur d'une image paternelle qu'elle n'ose affronter de peur de subir sa malédiction (II, i, 20-23). Plutôt que de l'encourir, elle préfêre utiliser la ruse et le meurtre.

Les rapports de Beatrice et d'Alsemero changent brutalement avec le mariage. La jeune fille ardemment désirée, parce qu'il la suppose vierge, devient un objet de méfiance dès que Jasperino lui fait part de ses soupçons :

Oh were she the sole glory of the earth, Had eyes that could shoot fire into kings' breasts, And touch'd, she sleeps not here !»

$$
\text { (IV, ii, 105-107). }
$$

En épousant Alsemero, Beatrice s'est donné un maître qui peut se révéler un impitoyable bourreau ; elle le sait et semble trouver cela naturel : s'il découvre pendant la nuit de noces qu'elle n'est pas vierge

He cannot but in justice strangle me As I lie by him

$$
\text { (IV, i, 14-15). }
$$

Soulignonscetéloquent "but in justice" : elle reconnaîtavoir méritéla mort dans la mesure où elle a enfreint le code de morale sexuelle de son milieu social.

Dans le Changeling, la peinture des rapports sociaux n'est certes pas ce qui intéresse les dramaturges au premier chef; il n'empêche qu'ils ont esquissé à l'arrière-plan le tableau d'une société fortement hiérarchisée, où le comportement de l'aristocratie est conditionné par un code $d$ "honneur" qu'aucun des personnages ne songe à mettre en question. Les rapports de domination, à l'intérieur de cette société, infléchissent inévitablement les rapports affectifs ; la peur ou la méfiance que les personanges éprouvent les uns à l'égard des autres détruit ou affaiblit les sentiments les plus instinctifs, les plus naturels. (Dans les comédies de Middleton et de certains de ses contemporains, c'est l'appât du gain qui joue le rôle d'agent destructeur). Mais dans le drame de Beatrice tel qu'il nous est présenté par les auteurs, c'est sa propre violence à l'égard d'Alonzo qui la sépare de celui qu'elle croit désormais aimer, cet Alsemero qui lui reproche, au cours de la dernière scène, d'avoir osé franchir «this dangerous bridge of blood» (vers 81); c'est son propre sentiment de honte qui l'éloigne de son père au moment de la mort : « $O$ come not 
near me, sir, I shall defile you» (vers 149) : elle est devenue étrangère à elle-même comme elle est devenue étrangère aux siens. Il convient maintenant d'analyser la forme d'aliénation qui, étroitement liée à celles que nous avons jusqu'ici rencontrées, donne au changeling sa pleine dimension morale. On qualifie souvent d'aliénant tout ce qui, parole ou acte, vise à transformer quelqu'un d'autre en instrument, à le traiter comme un simple objet, une simple marchandise, à le dépouiller ne serait-ce que d'une part de son humanité.

Ce qui différencie Isabella d'Alibius ou de Lollio, c'est que pour elle les pensionnaires de l'asile d'aliénés restent des hommes, et ceci l'empêche de trouver "mirth in madness" (III, iii, 27) ; un peu plus loin, (275-276), elle ironise :

$Y^{\prime}$ have a fine trade on't,

Madmen and fools are a staple commodity.

Alibius, lui, s'enrichit sans vergogne à leurs dépens, administre volontiers à son profit la fortune des aliénés (IV, iii, 209-210), et gagne à l'occasion un peu d'argent en les donnant en spectacle.

Mais il y a d'autres façons, plus banales, de réduire un être humain à l'état de "staple commodity"; par deux fois, Beatrice s'efforce de transformer en instruments à sa convenance les serviteurs qui vivent autour d'elle, en utilisant le pouvoir de l'argent. Parce que De Flores est pauvre, «Belike his wants are greedy» (II, ii, 125), elle l'imagine prêt à mettre sa force et son poignard au service de celle qui jusqu'ici l'a accablé de mépris et d'injures, à devenir un tueur docile et discret - etceci pour une somme qu'elle ne prend même pas la peine de préciser. Elle ignore encore qu'on ne cherche pas à corrompre autrui sans se corrompre soi-même, qu'on ne tente pas de faire d'un homme l'instrument d'un crime sans risquer de devenir son esclave. Elle croit acheter un tueur, et c'est son corps qu'elle vend. Pas de possession sans aliénation : c'est la leçon qui se dégage de l'affrontement dont elle sort vaincue à la fin du troisième acte.

On remarquera que la même leçon se dégage de Volpone où Ben Jonson, jouant en virtuose des divers sens de "possession", "possessed" et "dispossessed", montre à quel point celui qui s'attache à l'excès aux biens matériels est en fait possédé par eux en vertu d'une sorte de possession démoniaque. Apparemment, Beatrice n'a pas compris cette leçon, puisqu'ayant perdu sa virginité, elle manœuvre pour acheter celle de Diaphanta, car, pense-t-elle, «'tis a niece piece / Gold cannot purchase» (IV,i, 54-55), et, en effet, pour mille ducats, la servante accepte volontiers de se substituer à sa maîtresse dans le lit conjugal. Ayant fait de Diaphanta une sorte de prostituée, Beatrice s'étonne un peu naïvement qu'elle y prenne du plaisir au point d'en oublier l'heure : «This whore forgets herself» (V, i, 23). Grâce à De Flores, la mort interviendra une nouvelle fois dans ce marché douteux où l'argent et l'amour - si c'est bien le mot qui convient - servent de monnaie d'échange. Sinistre marché de dupes où Diaphanta paie de sa vie et l'amour et l'argent, et dont Alsemero ne sort 
pas grandi : sa nuit de noces est l'épilogue grotesque des tests de virginité qu'il a imposés à sa femme ; il est ridicule, parce qu'il a cru habile de la traiter en objet d'expérimentation, en cobaye, oubliant que le respect de soi-même implique le respect de l'Autre. Dans le Changeling, farce et tragédie se rejoignent pour rappeler la même vérité. Aberration mentale, monstruosité affective, produit des circonstances sociales et familiales, le meurtre d'Alonzo révèle que pour Beatrice on peut se débarrasser d'un homme comme d'un objet inutile et gênant. Au sujet de ce meurtre, elle ne se pose pas de questions morales; elle agit exactement comme Bianca, autre création de Middleton, qui, dans Women Beware Women, essayant d'obtenir du duc, son amant, qu'il la débarrasse de son mari, désigne ce dernier par cette périphrase : «the former thing» WBW, IV, i, 112).

Mais, comme l'avait déjà montré l'auteur de Macbeth, tuer un hommen'est pas s'en libérer. C'est aussi ce que nous dit Sartre, en un autre temps et un autre langage :

Celui qui, une fois, aété pour autrui est contaminédans sonêtre pour le restant de ses jours, autrui fût-il entierement supprimé : il ne cessera de saisir sa dimension d' être-pour-autrui comme une possibilité permanente de son être. Il ne saurait reconquérir ce qu'il a aliéné ; il a même perdu tout espoir d' agir sur cette aliénation dansla tombe... La mort de l' autre me constitue comme objet irrémédiable, exactement comme ma propre mort». (L'Etre et le Néant, p. 463).

«Nousmourronstous, c' est la seule aliénation sérieuse», écrit Ionesco dans Tueur sans gages; $c$ 'est là le point de vue d'un athée de notre siècle. Dans l'univers chrétien du Changeling, il y a une forme d'alinéation encore plus terrible que la mort, et le texte de la dernière scène la mentionne explicitement : c'est la damnation, perte irrévocable de l'amour de Dieu.

Affirmée ou contestée, la valeur de la raison humaine est au centre du débat intellectuel de la Renaissance. Les sceptiques comme Montaigne en font ressortir les limites ; d'autres, plus optimistes, comme Juste Lipse ou Richard Hooker tendent à faire confiance à la raison, conçue essentiellement comme la faculté qui nous permet de vivre en accord avec la morale et la volonté de Dieu. Le Changeling nous présente un monde où l'homme est victime de multiples aliénations, privé de jugement, perturbé dans son affectivité, mû par le désir et la violence; il ne respecte ni chez autrui ni en lui-même l'image du Créateur. Le pessimisme de cette vision reflète en partie l'inquiétude provoquée, au début du XVIIe siècle, par certains aspects nouveaux de l'éternelle exploitation de l'homme par l'homme ; il reflète sans doute d'abord la pensée calviniste, et son insistance sur la dépravation innée de la nature humaine. 